\title{
Automated Interpretation of Cardiac Arrhythmias
}

\section{Design and Evaluation of a Computerized Model}

\author{
Lorenzo A. DiCarlo, MD, Dongping Lin, PhD, \\ and Janice M. Jenkins, PhD
}

\begin{abstract}
Historically, the development of computerized models that utilize the deductive methods used by clinicians for the interpretation of cardiac arrhythmias have been limited by the absence of a consistently reliable means of detecting atrial activation. In this study, a theoretical model was developed with a hierarchical organization of problem-solving strategies utilizing automated analysis of atrial activation from a commercially available esophageal pill electrode and ventricular activation from a simultaneously recorded surface electrocardiographic lead. The theoretical model was then tested in 21 patients with 1 or more of 28 distinct supraventricular and ventricular arrhythmias. Of the 641 individual cardiac cycles analyzed, $636(99.2 \%)$ were correctly identified. The accuracy of a contextual, that is, more comprehensive, interpretation of consecutive cardiac cycles was 638/641 (99.5\%). The following cardiac arrhythmias were identified: sinus rhythm, sinus bradycardia, atrial premature depolarizations, atrial flutter, and supraventricular tachycardias with normal and aberrant ventricular conduction, first-degree and second-degree heart block; junctional escape, junctional rhythm, idioventricular rhythm, ventricular prematurc depolarization, and ventricular tachycardia with and without retrograde activation; atrial bigeminy, atrial trigeminy, atrial couplets, ventricular bigeminy, ventricular trigeminy, and ventricular couplets. This study represents the first computerized model ever developed to incorporate the morphology and timing of atrial activation with the morphology and timing of ventricular activation for arrhythmia diagnosis. Such modeling appears to be capable of achieving accurate interpretation of spontaneous, complex clinical cardiac arrhythmias and atrioventricular relationships. Key words: computer-assisted diagnosis, arrhythmia, esophageal electrode.
\end{abstract}

From the Department of Electrical Engineering and Computer Science, School of Engineering, and School of Medicine. University of Michigan, Ann Arbor, the Michigan Heart and Vascular Institute, and the Cardiac Electrophysiology Laboratory, St. Joseph Mercy Hospital, Ann Arbor. Michigan.

Partially supported by a grant from the Whitaker Foundation, NSF grant Nos. ECS-8351215 and ECS-8506494, and a grant from the office of the Vice President for Research at the University of Michigan.

Reprint requests: Lorenzo DiCarlo, $\mathrm{MD}$, Reichert Heath Building R-3003, Catherine McAuley Health Center, P.O. Box 994, Ann Arbor, MI 48106.
The clinical interpretation of cardiac arrhythmias using surface electrocardiography requires an incorporation of the temporal order of atrial and ventricular depolarizations with an analysis of atrioventricular and ventriculoatrial relationships. Historically. the development of automated systems that utilize such methods has had limited success. ${ }^{1-9}$

Arrhythmia diagnosis requires the determination of individual rates and morphologies of serial atrial 
and serial ventricular activations, the sequence of atrial and ventricular activation, and the comparisons of the interval between atrial and ventricular depolarization with the interval between ventricular and atrial depolarization to infer atrioventricular and ventriculoatrial relationships. Arriving at a diagnosis, however, may not necessarily mandate a systematic assessment of all available variables in a consistent and unbiased manner. For example, analysis of an isolated atrial or ventricular event might require integration of its occurrence with intervening events since the last sinus rhythm cycle alone. In other cases, analysis of a similar event may depend upon events prior to the last sinus rhythm cycle (Figs. I and 2).

A theoretical model was designed that, for the first time, examines and interprets the morphology and timing of atrial as well as ventricular events in the cardiac cycle. At the initial level of data organization, atrial-atrial, ventricular-ventricular, atrial-ventricular, and ventricular-atrial intervals and atrial and ventricular morphologies were identified and classified, providing a single cardiac cycle diagnosis. In a second level of more complex organization, a hier-

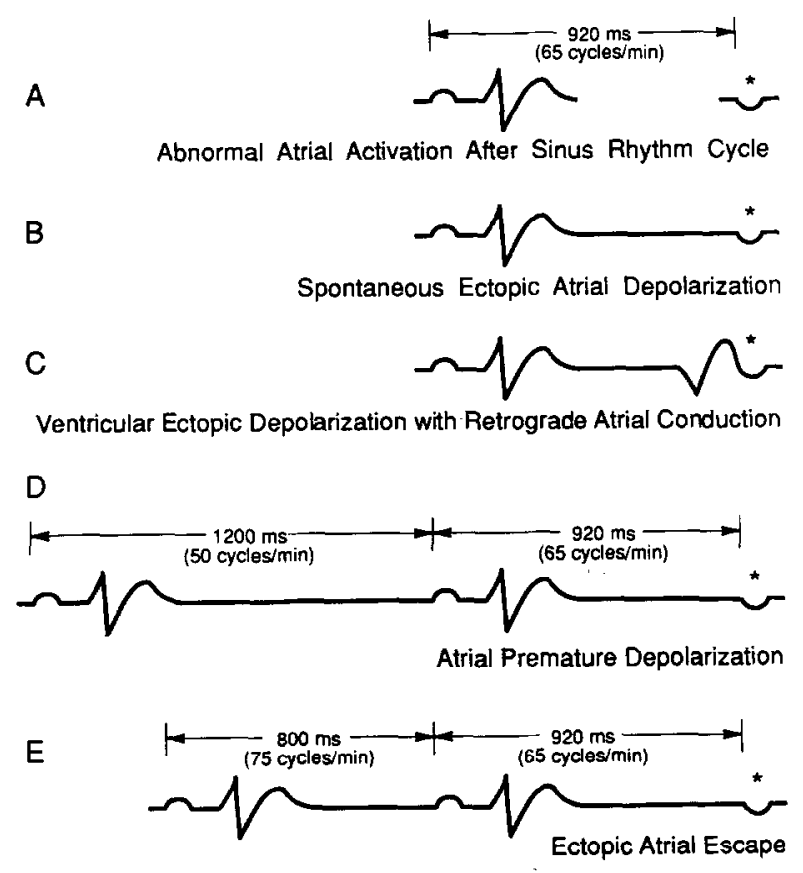

Fig. 1. Interpretation of an atrial depolarization $\left({ }^{*}\right)$ having an abnormal morphology when compared to sinus rythm (A). (B, C) Events intervening since the last sinus rhythm cycle determine whether it is an ectopic atrial depolarization that is spontaneous or due to retrograde conduction. (D, E) Events prior to the last sinus rhythm cycle determine whether the ectopic spontaneous atrial depolarization is premature or represents an escape mechanism:

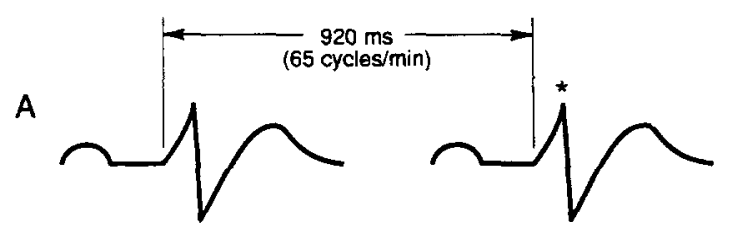

Normal Ventricular Activation After Sinus Rhythm Cycle
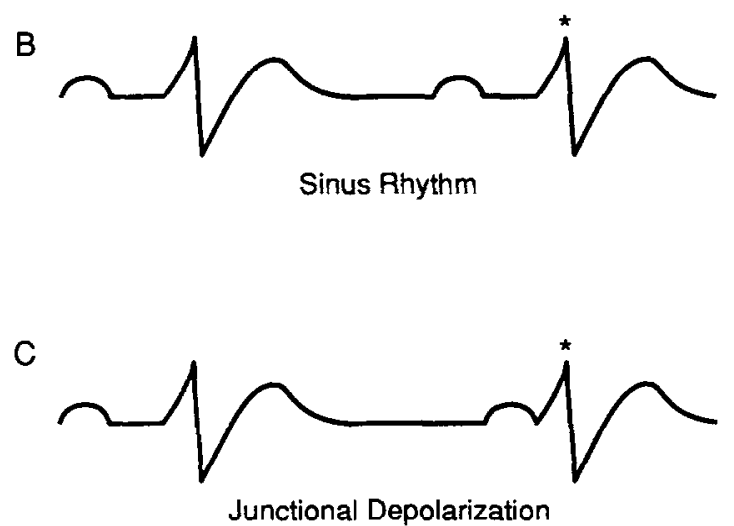

Fig. 2. Interpretation of a ventricular depolarization (*) having a normal morphology when compared to sinus rhythm (B). (B, C) Intervening events since the last sinus rhythm cycle determine whether it is spontaneous or due to anterograde atrial conduction.

archy was developed to determine the relationship of the individual cardiac cycle to the cardiac cycles that preceded it. In this second level of hierarchical organization, the diagnosis ascribed to the single cardiac cycle under analysis was incorporated with those of the eight preceding cardiac cycles to derive a contextual, that is, more comprehensive, diagnosis. This theoretical model was then tested in 21 patients with 1 or more of 28 different supraventricular or ventricular arrhythmias.

\section{Materials and Methods}

\section{Theoretical Model}

A block diagram of the five major components of the model is presented in Figure 3 . The components include: (1) data acquisition; (2) atrial and ventricu-

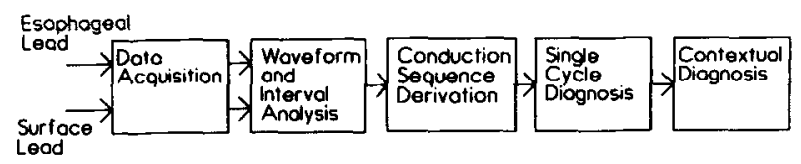

Fig. 3. Block diagram of the system consisting of five subsystems: data acquisition, waveform and interval analysis, conduction sequence derivation, single cycle diagnosis, and contextual diagnosis. 
lar waveform and interval analysis; (3) derivation of order of conduction sequence: (4) derivation of a five-digit, single cardiac cycle diagnostic code determined for each cardiac cycle; and (5) integrated interpretation of sequential cardiac cycles (contextual analysis).

Data Acquisition. A two-channel ambulatory or telemetry electrocardiographic system is used for simultaneous recording of atrial electrograms from an esophageal pill electrode and ventricular electrograms from surface electrocardiogram (ECG) lead II using previously described methods. ${ }^{10-13}$ After analog-to-digital conversion, the electrograms are analyzed.

Atrial and Ventricular Electrogram Waveform and Interval Analysis. Each cardiac cycle is analyzed for five features: (I) correlation waveform analysis of the atrial electrogram $\left(\mathrm{CC}_{\mathrm{a}}\right)$; (2) correlation waveform analysis of the ventricular electrogram $\left(C_{\mathrm{v}}\right)$ and interval determination between (3) two consecutive atrial depolarizations (AA); (4) atrial and subsequent ventricular depolarization (AV); and (5) two consecutive ventricular depolarizations (VV).

Waveform Analysis. In preprocessing prior to automated analysis, an atrial depolarization is selected from a normal sinus rhythm cycle as a normal atrial template. A ventricular depolarization from the samc cardiac cycle is selected as a normal ventricular template. The method of waveform analysis of subsequent cardiac cycles is a comparison of each subsequent atrial depolarization with the atrial template and each subsequent ventricular depolarization with the ventricular template, respectively, using correlation waveform analysis. ${ }^{11,14,15}$ This method of analysis has been traditionally applied to surface ECGs, ${ }^{14,15}$ and more recently to intracardiac electrograms. ${ }^{16-20}$

The following equation is used to calculate the correlation coefficient:

$$
\rho=\frac{\sum_{i=1}^{M}\left(T_{i}-\mu_{T}\right)\left(X_{i}-\mu_{X}\right)}{\sqrt{\sum_{i=1}^{M}\left(T_{i}-\mu_{T}\right)^{2} \sum_{i=I}^{M}\left(X_{i}-\mu_{X}\right)^{2}}}
$$

where

$$
\begin{aligned}
M & =\text { the number of points in the template; } \\
T_{i} & =\text { the template points; } \\
X_{i} & =\text { signal points to be processed; and } \\
\mu & =\text { the signal or template average. }
\end{aligned}
$$

The correlation coefficient $(\rho)$ represents a mea- sure of similarity between a signal and a template in the range $-1<\rho<+1$, where +1 indicates a perfect match. In this system, a depolarization with $\rho \geq$ 0.95 is considered a match, that is, normal; those falling below this value are classified as abnormal.

Interval Analysis. Interval analysis is performed by comparing each incoming $\mathrm{AA}, \mathrm{AV}$, and $\mathrm{VV}$ interval with the AA, AV, and VV intervals determined during the normal sinus rhythm cycle. In this study, the upper and lower limits of the AA and VV intervals were set at $\pm 15 \%$ of the template normal sinus rhythm cycle. Intervals of the cardiac cycle being analyzed are classified into three categories. If an interval falls below the lower limit of its normal range, it is classified as short; if above, it is classified as long. If the interval is between the lower and upper limits, it is classified as normal.

Single Cardiac Cycle Code. For each cardiac cycle, a five-digit single cardiac cycle code is determined for the five analyzed features (Fig. 4). The digits assigned for atrial morphology and ventricular morphology are either 0 (abnormal) or 1 (normal). The digits assigned for the AA, AV, and VV intervals are 0 (short), 1 (normal), or 2 (long).

Definitions of Normal $A A, A V$, and VV Intervals During Sinus Rhythm. During sinus rhythm at rates of 60-100 cycles/min, the upper and lower limits of a normal AA and a normal VV interval are 1.15 and 0.85 times the template sinus cycle $\mathrm{AA}$ and $\mathrm{VV}$ intervals. The lower limit of a normal AV interval is 0.8 times the template sinus cycle AV interval or $100 \mathrm{~ms}$ if the latter value is greater. The upper limit of a normal AV interval is 1.2 times the last sinus cycle AV interval or $220 \mathrm{~ms}$ if the latter value is greater.

Definitions of Atrioventricular Conduction. In cardiac cycles where an atrial depolarization is not followed

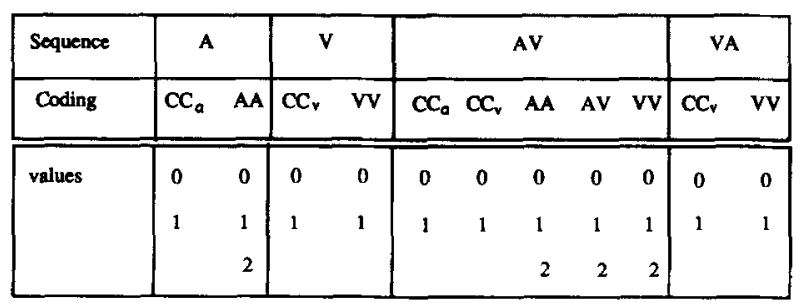

Fig. 4. The coding method for all sequences. Correlation waveform analysis of the atrium $\left(\mathrm{CC}_{\mathrm{a}}\right)$ and the ventricle $\left(C_{v}\right)$ can be 0 (abnormal) or 1 (normal). Consecutive atrial depolarizations (AA) and the atrioventricular interval (AV) can be 0 (short), l (normal), or 2 (long). Two consecutive ventricular depolarizations (VV) can be 0 (short), 1 (normal), or 2 (long) for the AV sequence; and 0 (short) or 1 (long) for the ventricular (V) and ventriculoatrial (VA) sequences. 
by a ventricular depolarization, $\mathrm{CC}_{\mathrm{v}}$ and $\mathrm{AV}$ and $\mathrm{VV}$ intervals cannot be determined. The single cardiac cycle diagnostic code contains digits for only two diagnostic features $\left(\mathrm{CC}_{\mathrm{a}}\right.$ and $\mathrm{AA}$ interval), and the atrial depolarization is defined as nonconducted.

In this theoretical model, an atrial depolarization followed by a ventricular depolarization with an AV interval greater than $360 \mathrm{~ms}$ is also defined as a nonconducted atrial depolarization, even though all five diagnostic features can be derived from such a cardiac cycle.

If two consecutive atrial depolarizations occur and a ventricular depolarization does not precede the second atrial depolarization, determination of atrioventricular conduction is defined by four features and is summarized in Table 1.

Definitions of Ventriculoatrial Conduction. In cardiac cycles where a ventricular depolarization is not preceded by an atrial depolarization, a sixth feature for the single cardiac cycle is utilized: the ventriculoatrial (VA) interval. It is measured from the ventricular depolarization to the next atrial depolarization which occurs. Ventriculoatrial conduction is defined to have occurred if the $\mathrm{CC}_{\mathrm{a}}$ of the atrial depolarization is 0 (abnormal) and the measured VA interval is at least $100 \mathrm{~ms}$ but $<400 \mathrm{~ms}$.

If two ventricular depolarizations occur consecutively without an intervening atrial depolarization, retrograde atrial conduction by the first ventricular depolarization is defined by five features: (1) the VV interval of the first ventricular depolarization is 0 (shorter than the normal VV interval during sinus rhythm), (2) the $\mathrm{CC}_{\mathrm{a}}$ of the subsequent atrial depolarization is 0 (abnormal), (3) the AA interval of the atrial depolarization is 1 (unchanged) or 0 (shorter than the normal AA interval during sinus rhythm),
(4) the VA interval measured from the first of the two consecutive ventricular depolarizations may be 0,1 , or 2 (within $100-400 \mathrm{~ms}$ ), (5) the VA interval measured from the second of the two consecutive ventricular depolarizations is $<100 \mathrm{~ms}$. Figure 4 shows the coding scheme for each of the possible sequences ( $\mathrm{A}, \mathrm{V}, \mathrm{AV}$, or VA).

Single Cardiac Cycle Diagnosis. The construction of cardiac cycle codes from these rules results in 122 possible diagnostic statements for single cardiac cycle classification. Ladder diagrams for all 122 codes are given in the Appendix. Single cycle diagnoses include: atrial premature depolarization, atrial ectopic depolarization, atrial escape, sinus slowing (bradycardia depolarization), normal ventricular premature depolarization, junctional escape, ectopic ventricular premature depolarization, ventricular escape, and ventricular fusion. Each atrial depolarization is further characterized as conducted or nonconducted, conducted with AV delay (first-degree AV block), and/or conducted with normal or aberrant ventricular depolarization. Each normal or ventricular ectopic depolarization that is not preceded but followed by an atrial depolarization is further characterized as occurring with or without retrograde atrial activation. (Table 2.)

Integrated Interpretation of Sequential Cardiac Cycles (Contextual Diagnosis). Single cycle diagnoses of the eight most current cardiac cycles are incorporated to derive a contextual diagnosis. Possible contextual diagnoses include sinus, atrial, junctional, or idioventricular bradycardias; first-degree and second-degree AV block; atrial bigeminy, atrial trigeminy, atrial couplet, ventricular bigeminy, ven-

Table 1. Definitions of Atrioventricular Conduction and Block of the First of Two Consecutive Atrial Depolarizations Without an Intervening Ventricular Depolarization

\begin{tabular}{|c|c|c|c|c|}
\hline A $_{1}$ Interval* & $\mathbf{A}_{1} \mathbf{A}_{2}$ Interval* & $\mathrm{CC}_{\mathrm{Al}}$ & $\mathbf{A}_{2} \mathbf{V}$ & $\mathbf{A}_{\mathbf{l}}$ \\
\hline Normal & Normal & - & - & Nonconducted \\
\hline Normal & Short & $\begin{array}{l}\text { Normal } \\
\text { Abnormal }\end{array}$ & $\begin{array}{l}\text { Unchanged or Long } \\
\text { Short, unchanged, or long }\end{array}$ & $\begin{array}{l}\text { Conducted } \\
\text { Conducted }\end{array}$ \\
\hline Normal & Long & - & - & Nonconducted \\
\hline Short & Normal & - & - & Nonconducted \\
\hline Short & Short & $\begin{array}{l}\text { Normal } \\
\text { Abnormal }\end{array}$ & $\begin{array}{l}\text { Unchanged or long } \\
\text { Short, unchanged, or long }\end{array}$ & $\begin{array}{l}\text { Conducted } \\
\text { Conducted }\end{array}$ \\
\hline Short & Long & - & - & Nonconducted \\
\hline Long & Normal & - & - & Nonconducted \\
\hline Long & Short & $\begin{array}{l}\text { Normal } \\
\text { Abnormal }\end{array}$ & $\begin{array}{l}\text { Unchanged or long } \\
\text { Short, unchanged, or long }\end{array}$ & $\begin{array}{l}\text { Conducted } \\
\text { Conducted }\end{array}$ \\
\hline Long & Long & - & - & Nonconducted \\
\hline
\end{tabular}

$A=$ atrial depolarization with ventricular depolarization preceding $A_{1} ; A_{1}=$ first of two consecutive premature depolarizations without an intervening ventricular depolarization; $A_{2}=$ second of the two consecutive premature depolarizations; $C C_{A_{1}}=$ atrial morphology of $A_{1}$ when compared to the preprocessed atrial template determined during sinus rhythm; $A_{1} V=$ the measured atrioventricular interval from $A_{1}$ to the next observed ventricular depolarization. *When compared to the last AA interval that occurred during sinus rhythm. 
Table 2. Table of Single Cardiac Cycle Classes

\begin{tabular}{|c|c|c|}
\hline Class & Type & Characteristics \\
\hline I & Normal & $\begin{array}{l}\mathrm{CC}_{\mathrm{a}}, \mathrm{CC}_{\mathrm{v}} \text {, and at least two normal } \\
\text { intervals }\end{array}$ \\
\hline 2 & Aberrant & $\begin{array}{l}\text { Sinus depolarization with aberrant } \\
\text { conduction }\end{array}$ \\
\hline 3 & AV delay & Sinus depolarization with $\mathrm{AV}$ delay \\
\hline 4 & Blocked & Blocked sinus depolarization \\
\hline 5 & APD & $\begin{array}{l}\text { Atrial premature depolarization with or } \\
\text { without conduction }\end{array}$ \\
\hline 6 & $\mathrm{JP}$ & $\begin{array}{l}\text { Junctional premature depolarization with } \\
\text { or without retrograde }\end{array}$ \\
\hline 7 & VPD & $\begin{array}{l}\text { Ventricular premature depolarization with } \\
\text { or without retrograde }\end{array}$ \\
\hline 8 & SS & $\begin{array}{l}\text { Sinus slowing with or without } \\
\text { conduction }\end{array}$ \\
\hline 9 & $\mathrm{AE}$ & $\begin{array}{l}\text { Atrial ectopic depolarization with or } \\
\text { without conduction }\end{array}$ \\
\hline 10 & $\mathrm{JE}$ & $\begin{array}{l}\text { Junctional escape with or without } \\
\text { retrograde }\end{array}$ \\
\hline 11 & VE & $\begin{array}{l}\text { Ventricular escape with or without } \\
\text { retrograde }\end{array}$ \\
\hline 12 & NS & Nonspecific abnormal depolarization \\
\hline
\end{tabular}

$\mathrm{CC}_{\mathrm{a}}=$ atrial electrogram morphology; $\mathrm{CC}_{\mathrm{v}}=$ ventricular electrogram morphology.

tricular trigeminy, ventricular couplet, atrial tachycardia, atrial flutter, atrial fibrillation, ventricular tachycardia, ventricular flutter, and ventricular fibrillation.

Sustained supraventricular arrhythmias are then further characterized by their blocked or anterograde conduction pattern $(1: 1,2: 1,3: 1$, etc.), and sustained ventricular arrhythmias are characterized by the presence or absence of atrial retrograde conduction and the retrograde conduction pattern $(1: 1$, $2: 1,3: 1$, etc.).

To achieve this characterization four counters are employed. The first counter (AA) records the number of current sequential atrial depolarizations with short or long AA intervals. The sccond counter (VV) records the number of current sequential ventricular depolarizations with short or long VV intervals. The third counter (AV) records the number of atrioventricular activations that are recorded during a supraventricular bradycardia or tachycardia. The fourth counter (VA) records the number of ventriculoatrial activations detected during a ventricular bradycardia or tachycardia.

Contextual Diagnostic Definitions. Figure 5 shows a flow chart of the contextual diagnostic scheme. During the contextual diagnosis, a hierarchial priority scheme is employed. In this model, ventricular arrhythmias are sought first.

If the initial depolarization examined is an ectopic ventricular premature depolarization and is followed by five or more consecutive ventricular premature

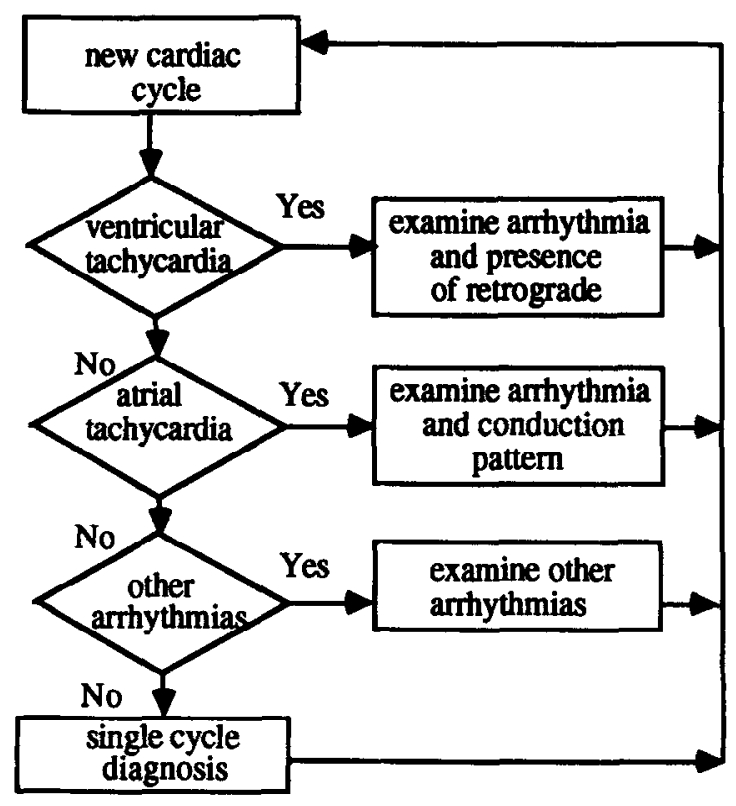

Fig. 5. Flow chart of contextual diagnosis showing the priority of arrhythmia diagnoses.

depolarizations, the contextual diagnosis is ventricular tachycardia, ventricular flutter, or ventricular fibrillation. The contextual diagnosis system examines the ventricular rate to classify the arrhythmia into ventricular fibrillation (more than 330 depolarizations per minute), ventricular flutter (240-330 depolarizations per minute), or ventricular tachycardia (100-240 depolarizations per minute). For ventricular fibrillation, the system reports ventricular fibrillation. For ventricular flutter or tachycardia, the system examines for retrograde atrial activation (abnormal $\mathrm{CC}_{\mathrm{a}}$ ) and records each such event using the ventriculoatrial activation counter. If the counter records zero, the system reports ventricular flutter without retrograde or ventricular tachycardia without retrograde. If the counter is not zero, the conduction pattern is calculated by dividing the last complete AA interval by the $\mathrm{VV}$ interval. If these intervals are equivalent, the arrhythmia is seen as $1: 1$ retrograde. If the AA interval is twice the current $\mathrm{VV}$ interval, there is $2: 1$ retrograde. Other retrograde patterns $(3: 1,4: 1$, etc.) are determined in the same manner.

If no ventricular tachycardia is present, the system searches for supraventricular tachycardia. If an atrial premature depolarization is followed by five or more consecutive atrial premature depolarizations, the contextual diagnosis system then examines the type of supraventricular tachycardia pattern present.

Atrial fibrillation is diagnosed if the atrial rate is greater than 330 cycles per minute. Atrial flutter is 
diagnosed if the atrial rate is $240-330$ cycles per minute. Supraventricular tachycardia is diagnosed if the atrial rate is between 140 and 240 cycles per minute. An atrial conduction counter indicates whether the dysrhythmia conducts to the ventricles. If so, the conduction pattern $(1: 1,2: 1,3: 1$, etc.) is calculated by dividing the last complete VV interval by the current AA interval. The supraventricular tachycardia and its conduction pattern $(1: 1,2: 1,3: 1$, etc. $)$ is then reported.

If there is no atrial or ventricular tachycardia, the system next examines for bradycardia. If the current depolarization is a sinus or atrial slowing depolarization and is followed by five or more such depolarizations in succession, the system reports a contextual diagnosis of bradycardia.

If atrial tachycardia, ventricular tachycardia, or bradycardia is not present, the system searches for three or more consecutive ventricular or atrial premature depolarizations by checking ventricular premature or atrial premature counters. If the number in either counter exceeds two, the consecutive ventricular or atrial premature depolarizations are reported along with the retrograde or anterograde conduction pattern.

Bigeminal and Trigeminal Rhythms. If none of the above is detected, the system examines the last four depolarizations for atrial and ventricular trigeminy or bigeminy. If there is an atrial premature depolarization (APD) for every two normal ventricular depolarizations in a repeated fashion (Normal-NormalAPD), the system reports atrial trigeminy. If the last atrial premature depolarization is conducted, a diagnosis of atrial trigeminy with conduction is given; otherwise, the diagnosis is atrial trigeminy without conduction. If there is an ectopic ventricular premature dcpolarization (VPD) for every two sinus depolarizations in a repeated fashion (Normal-NormalVPD), ventricular trigeminy is diagnosed. A diagnosis of ventricular trigeminy with retrograde conduction, or ventricular trigeminy without retrograde conduction is reported depending on whether or not the last ventricular ectopic depolarization causes retrograde atrial activation. Atrial and ventricular bigeminy are diagnosed in similar fashion. Anterograde and retrograde conduction patterns are also diagnosed for atrial and ventricular bigeminy, respectively.

Couplets. Two consecutive atrial premature depolarizations or two atrial premature depolarizations with an intervening ventricular depolarization without retrograde activation will elicit a diagnosis of atrial couplet. The conduction pattern is determined by a counter that records the number of consecutive AV conductions of atrial premature depolarizations. Atrial couplet without conduction will be reported if the counter is zero; atrial couplet with $1: 1$ conduc- tion if the counter is 2 ; and atrial couplet with $2: 1$ conduction if the counter is 1 .

\section{Clinical Evaluation of the Theoretical Model}

\section{Data Acquisition}

After obtaining informed written consent, tape recorded data were acquired from patients in a coronary care unit utilizing an esophageal lead and a standard surface lead II. In this study, the esophageal electrograms were recorded from a bipolar pill electrode (Arzco Medical Electronics, Inc., Chicago, IL) swallowed by the patient and located posterior to the left atrium. ${ }^{10-13}$ The esophageal signal and surface lead were recorded with a bandwidth of $5-100 \mathrm{~Hz}$ and $0.05-100 \mathrm{~Hz}$, respectively. The $5 \mathrm{~Hz}$ low frequency cut-off applied to the esophageal signal eliminates low-frequency artifact such as respiration and cardiac motion. Recordings (15 minutes to 2 hours) were made from 21 patients who had 1 or more of 28 distinct arrhythmias. Data were digitized for computer processing using two-channel concurrent analog-to-digital conversion with a sampling rate of $1,000 \mathrm{~Hz}$ per channel. The data acquisition subsystem (Tecmar LabMaster, Solon, $\mathrm{OH}$ ) was interfaced to a personal computer. A software trigger (digital differentiator) was used for $\mathrm{P}$ wave and QRS waveform recognition.

A total of 29 passages, each containing 1 or more of 28 distinct arrhythmias, were tested. The passages to be processed were specifically chosen to contain a preponderance of abnormal cardiac cycles in order to assess the robustness of the detection system. Sinus rhythm cycles constituted only $25 \%$ of the cardiac cycles analyzed.

Each cardiac cycle and cardiac arrhythmia was evaluated and a diagnosis was given by a clinical cardiac electrophysiologist who was blinded to the results of the computerized analysis. The results were subsequently compared.

Figures 6 and 7 show two examples of computerprocessed passages. Figure 6 shows a recording and the results of the analysis from a patient who has a supraventricular tachycardia with $1: 1$ conduction with both onset of the tachycardia and spontaneous conversion. All ventricular depolarizations have normal morphologies. Cardiac cycles 2-5 are normal. Cycle 6 has long AA and VV intervals and is diagnosed as sinus slowing. Cycles 7 and 8 revert to normal. Subsequently, the patient develops a series of 


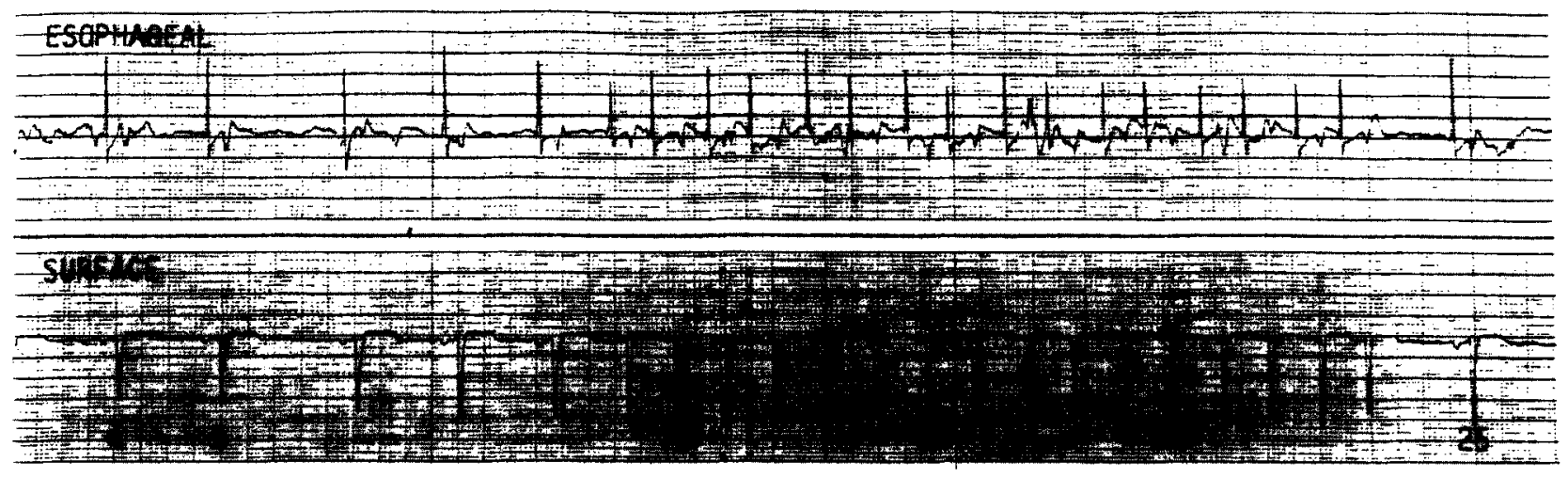

Cycle Seq Code Cycle Diagnosis

Contextual Diagnosis

$\begin{array}{rllll}4 & \text { AV } & 11111 & \text { Normal } & \text { NSR } \\ 5 & \text { AV } & 11111 & \text { Normal } & \text { NSR } \\ 6 & \text { AV } & 11212 & \text { Sinus slowing with cond. } & \text { Sinus slowing with cond } \\ 7 & \text { AV } & 11111 & \text { Normal } & \\ 8 & \text { AV } & 11111 & \text { Normal } & \\ 9 & \text { AV } & 11010 & \text { APD with cond } & \text { APD with cond. } \\ 10 & \text { AV } & 11010 & \text { APD with cond } & 1: 1 \text { Atrial couplet } \\ 11 & \text { AV } & 11010 & \text { APD with cond } & 3 \text { APDs with } 1: 1 \text { cond. } \\ 12 & \text { AV } & 11010 & \text { APD with cond } & 4 \text { APDs with } 1: 1 \text { cond. } \\ 13 & \text { AV } & 01010 & \text { APD with cond } & \text { 5 APDs with } 1: 1 \text { cond. } \\ 14 & \text { AV } & 11010 & \text { APD with cond } & 1: 1 \text { SVT } \\ 15 & \text { AV } & 11010 & \text { APD with cond } & 1: 1 \text { SVT } \\ 16 & \text { AV } & 11010 & \text { APD with cond } & 1: 1 \text { SVT } \\ 17 & \text { AV } & 11010 & \text { APD with cond } & 1: 1 \text { SVT } \\ 18 & \text { AV } & 11010 & \text { APD with cond } & 1: 1 \text { SVT } \\ 19 & \text { AV } & 11010 & \text { APD with cond } & 1: 1 \text { SVT } \\ 20 & \text { AV } & 11010 & \text { APD with cond } & 1: 1 \text { SVT } \\ 21 & \text { AV } & 11010 & \text { APD with cond } & 1: 1 \text { SVT } \\ 22 & \text { AV } & 11010 & \text { APD with cond } & 1: 1 \text { SVT } \\ 23 & \text { AV } & 11010 & \text { APD with cond } & 1: 1 \text { SVT } \\ 24 & \text { AV } & 11010 & \text { APD with cond } & 1: 1 \text { SVT } \\ 25 & \text { AV } & 11111 & \text { Normal } & \end{array}$

Fig. 6. Supraventricular tachycardia with $1: 1$ conduction showing both onset and conversion. This patient has normal sinus rhythm (NSR) at the beginning of the passage (cycles 1-5) followed by a sinus slowing (cycle 6) and an initiation of supraventricular tachycardia at cycle 9. Spontaneous conversion occurs at cycle 25 . All ventricular depolarizations are similar. $\mathrm{APD}=$ atrial premature depolarization; $\mathrm{AV}=$ atrioventricular; cond $=$ conduction; seq $=$ sequence.

atrial premature depolarizations that conduct to the ventricles. At the beginning of the tachycardia (cycles 9 and 10), the system delivers a contextual diagnosis of atrial couplet with $1: 1$ conduction. For the next three cycles the diagnosis is 3,4 , and 5 consecutive atrial premature depolarizations with $1: 1$ conduction. After six consecutive conducted atrial premature depolarizations occur, the rhythm diagnosis is 1:1 supraventricular tachycardia, which continues to be reported until conversion occurs (cycle 25).
Figure 7 shows a recording and the results of the analysis from a patient who has ventricular couplets with retrograde atrial activation. The couplets in this example occur at cycles 5,6 and 14, 15. All ventricular premature depolarizations in these couplets have retrograde atrial activation with the abnormal atrial depolarizations that follow each ventricular premature depolarization. The system diagnoses pairs of ventricular premature depolarizations in the individual cycle stage and correctly makes the contextual 


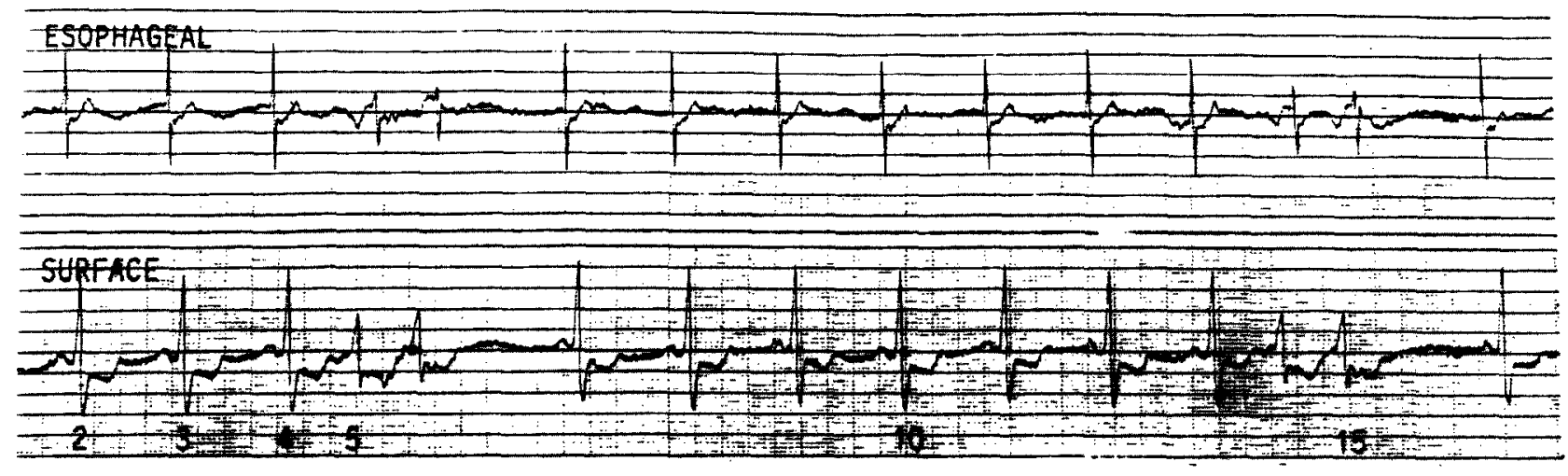

\section{Cycle Seq Code Cycle Diagnosis Contextual Diagnosis}

$\begin{array}{rlrll}2 & \text { AV } & 11111 & \text { Normal } & \\ 3 & \text { AV } & 11111 & \text { Normal } & \\ 4 & \text { AV } & 11111 & \text { Normal } & \text { NSR } \\ 5 & \text { VA } & 00 & \text { VPD with retrograde } & \text { VPD with retrograde } \\ 6 & \text { VA } & 00 & \text { VPD with retrograde } & \text { V couplet with 1:1 retro. } \\ 7 & \text { AV } & 11212 & \text { Sinus slowing with cond. } & \text { Sinus slowing with cond. } \\ 8 & \text { AV } & 11111 & \text { Normal } & \\ 9 & \text { AV } & 11111 & \text { Normal } & \\ 10 & \text { AV } & 11111 & \text { Normal } & \text { NSR } \\ 11 & \text { AV } & 11111 & \text { Normal } & \text { NSR } \\ 12 & \text { AV } & 11111 & \text { Normal } & \text { NSR } \\ 13 & \text { AV } & 11111 & \text { Normal } & \text { NSR } \\ 14 & \text { VA } & 00 & \text { VPD with retrograde } & \text { VPD with retrograde } \\ 15 & \text { VA } & 00 & \text { VPD with retrograde } & \text { V couplet with } 1: 1 \text { retro. } \\ 16 & \text { AV } & 11212 & \text { Sinus slowing with cond. } & \text { Sinus slowing with cond. }\end{array}$

Fig. 7. This patient has ventricular premature depolarizations (VPD) and ventricular (V) couplets, (cycles 5 and 6 , and 14 and 15) with ventricular atrial (VA) or retrograde (retro) activation. Each VPD is diagnosed in the single cycle stage and the contextual diagnosis of the couplet appears upon the second VPD of each pair. Abbreviations same as in Figure 6.

diagnosis of ventricular couplet with $1: 1$ retrograde activation.

\section{Results}

\section{Single Cardiac Cycle Diagnostic Code}

For the 641 individual cardiac cycles of the 29 passages analyzed, the diagnostic accuracy was $99.2 \%$ (636 of 641). There were no false positives (abnormal cardiac cycles classified as normal). All five errors were abnormal cardiac cycles that were misclassified. Two of these five errors were corrected by contextual diagnosis.

\section{Contextual Diagnosis}

The accuracy of the contextual diagnosis of the recorded cardiac arrhythmias analyzed was $99.5 \%$ (638 of 641 diagnostic statements).

The following types of cardiac arrhythmias were identified: normal sinus rhythm, sinus rhythm with aberrant ventricular activation; sinus rhythm without ventricular activation (blocked sinus cycle); atrial premature depolarizations with normal or aberrant ventricular activation, atrial premature depolarizations without ventricular activation (blocked); ventricular premature depolarizations with or without retrograde atrial activation; sinus slowing with or without ventricular activation; junctional escape or junctional rhythm with or without retrograde atrial activation; ectopic ventricular escape or idioventricular rhythm with or without retrograde atrial activa- 
tion; atrial bigeminy; atrial trigeminy; atrial couplets; ventricular bigeminy; ventricular trigeminy; ventricular couplets; ventricular tachycardia; supraventricular tachycardia; atrial flutter; first-degree atrioventricular block; and second-degree atrioventricular block.

\section{Discussion}

The absence of a consistently reliable means of detecting atrial activation using standard surface electrography has been one of the major obstacles to the implementation of automated methods for accurate arrhythmia diagnosis. Models developed to date have had to rely predominantly upon changes in the timing and morphology of ventricular electrograms to infer relationships between atrial and ventricular activation. As a result, they have had limited diagnostic accuracy. ${ }^{\text {I-9 }}$ For example, systems used currently for ambulatory, bedside, and intraoperative ECG monitoring are unable to accurately differentiate such elemental arrhythmias as atrial premature depolarizations, or supraventricular tachyarrhythmias with aberrant ventricular conduction from ventricular premature depolarizations or wide-complex tachycardias that are ventricular in origin.

Recently, the incorporation of surface ECG with the esophageal pill electrodes for the detection of atrial activity has been demonstrated to improve the accuracy of bedside cardiac arrhythmia interpreta-

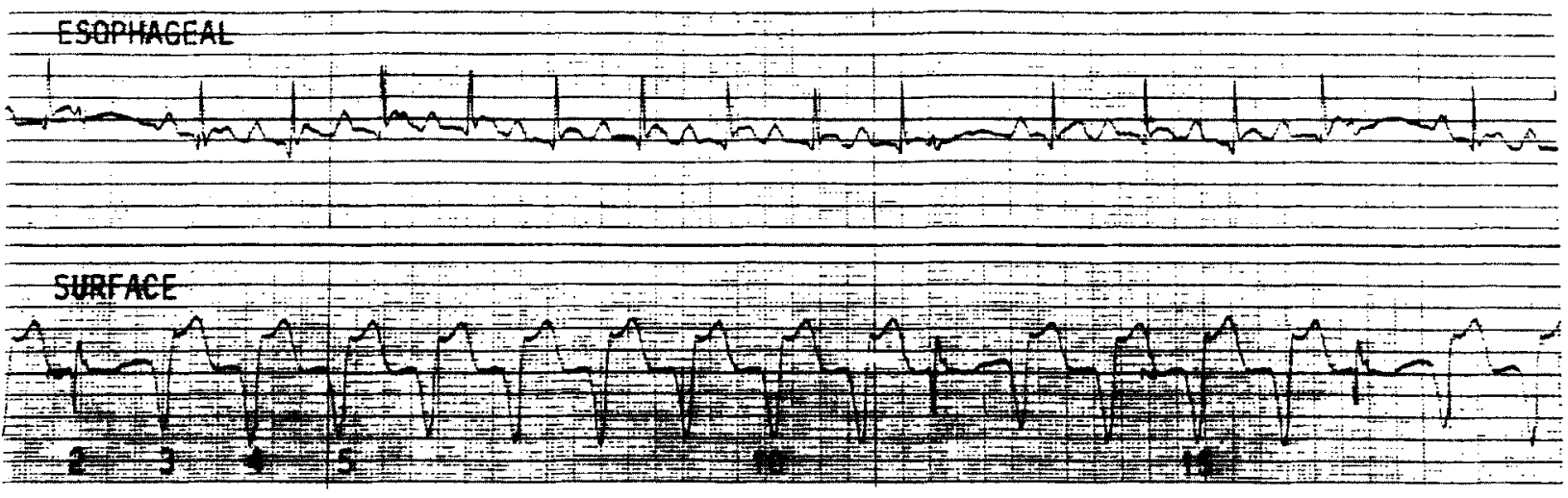

Cycle Seq Code Cycle Diagnosis Contextual Diagnosis

$\begin{array}{rrrll}2 & \text { AV } & 01020 & \text { APD with AV delay } & \text { APD with AV delay } \\ 3 & \text { VA } & 00 & \text { VPD with retrograde } & \text { VPD with retrograde } \\ 4 & \text { VA } & 00 & \text { VPD with retrograde } & \text { V couplet with } 1: 1 \text { retro. } \\ 5 & \text { VA } & 00 & \text { VPD with retrograde } & \text { 3 VPDs with } 1: 1 \text { retro. } \\ 6 & \text { VA } & 00 & \text { VPD with retrograde } & \text { 4 VPDs with } 1: 1 \text { retro. } \\ 7 & \text { VA } & 00 & \text { VPD with retrograde } & 5 \text { VPDs with } 1: 1 \text { retro. } \\ 8 & \text { VA } & 00 & \text { VPD with retrograde } & \text { VT with } 1: 1 \text { retro. } \\ 9 & \text { VA } & 00 & \text { VPD with retrograde } & \text { VT with } 1: 1 \text { retro. } \\ 10 & \text { VA } & 00 & \text { VPD with retrograde } & \text { VT with } 1: 1 \text { retro. } \\ 11 & \text { VA } & 00 & \text { VPD with retrograde } & \text { VT with } 1: 1 \text { retro. } \\ 12 & \text { V } & 10 & \text { J premature w/o retro. } & \text { V echo beat } \\ 13 & \text { VA } & 00 & \text { VPD with retrograde } & \text { VT with } 1: 1 \text { retro. } \\ 14 & \text { VA } & 00 & \text { VPD with retrograde } & \text { VT with } 1: 1 \text { retro. } \\ 15 & \text { VA } & 00 & \text { VPD with retrograde } & \text { VT with } 1: 1 \text { retro. } \\ 16 & \text { VA } & 00 & \text { VPD with retrograde } & \text { VT with } 1: 1 \text { retro. } \\ 17 & \text { V } & 10 & \text { J premature w/o retro. } & \text { V echo beat } \\ 18 & \text { VA } & 00 & \text { VPD with retrograde } & \text { VT with } 1: 1 \text { retro. }\end{array}$

Fig. 8. Ventricular tachycardia (VT) with $1: 1$ retrograde and echo beats. Cycles 2,12 , and 17 are mistakenly identified in the single cycle diagnostic scheme as junctional (J) premature depolarization without (w/o) retrograde conduction to the atrium. This is duc to their normal shape and short VV interval. They are, in fact, ventricular echo beats, and the latter two are recognized as such when examined by the contextual algorithm. Abbreviations same as in Figure 6. 
tion by clinicians. ${ }^{10-13}$ The results of this study suggest that the incorporation of similar data can provide a practical means of increasing the complexity and accuracy of automated ECG analysis. The addition of an esophageal atrial signal provides an effective feature for the analysis of the morphology, as well as the timing of atrial activation, thereby permitting the development of an artificial intelligence system for the accurate and comprehensive interpretation of individual cardiac cycles and supraventricular and ventricular arrhythmias.

The importance of contextual diagnosis in accurate arrhythmia analysis is emphasized by one of the limitations of this model. The single cardiac cycle analysis of this model cannot differentiate single atrial depolarizations with normal or prolonged AV conduction or aberrant conduction from single atrial depolarizations followed by a coincidental ventricular ectopic depolarization. There were two such cardiac cycles in a total of 641 analyzed cycles $(0.3 \%$ ) (Fig. 8). In addition, the single cardiac cycle diagnostic code could not detect ventricular echo beats because only the one or two most current depolarizations were considered during cardiac cycle coding. As a consequence, two ventricular "echo" depolarizations were misclassified as junctional depolarizations because of their proximity to the antecedent premature ventricular depolarization that was followed by retrograde atrial activation. The recognition of VA activation (which was accurate) precluded the recognition of the consequent AV conduction that followed. Despite this, the single cardiac cycle errors were corrected by the contextual diagnosis.

\section{Present and Future Applications}

Among the potential, practical applications of the type of model developed in this study are accurate automated interpretation of ambulatory ECG (Holter Monitor) recordings and improved automated analysis during intensive care or intraoperative ECG monitoring. Prolonged monitoring with esophageal electrodes has already demonstrated to be feasible. ${ }^{21}$ Further improvements and implementation of analogous models of arrhythmia analysis into devices such as implantible antitachycardia pacemakers and automatic cardioverter-defibrillators might provide more reliable discrimination of ventricular tachycardia and ventricular fibrillation from other, supraventricular tachyarrhythmias, and help prevent inappropriate device-patient interactions. ${ }^{22-24}$

Among possible improvements to be made in fur- ther models of arrhythmia analysis is the development of more streamlined analysis methods in order to decrease computational time. The possibility of decreasing computational demands for electrogram morphology analysis while maintaining the diagnostic accuracy of morphologic evaluation has been recently demonstrated. ${ }^{25,26}$ Further refinement of the contextual diagnosis system will be another important step in the development of efficient computerized dysrhythmia analysis. For example, future computerized modeling that incorporates strategies such as probability could increase the efficiency of the existing system. By limiting the total number of hypotheses that must be considered when individual cardiac cycles are incorporated into a contextual diagnosis, the total number of variables requiring analysis could also be decreased.

\section{Acknowledgment}

The authors wish to express their appreciation to Ms. Debbie Laird for preparation of the manuscript.

\section{References}

1. Pipberger HV, Fries ED, Taback L et al: Preparation of electrocardiographic data for analysis by digital electronic computer. Circulation 21:413, 1960

2. Staples IF, Gustafson JE, Balm GJ et al: Computer interpretation of electrocardiograms. Am Heart J 72: 351, 1966

3. Bonner RE, Schwetman HD: Computer diagnosis of the electrocardiogram II. Computer program for arrhythmia diagnosis. Comput Biomed Res 1:366, 1968

4. Bonner RE, Crevasse L, Ferrer MI et al: A new computer program for analysis of scalar electrocardiograms. Comput Biomed Res 5:629, 1972

5. Pryor TA, Lindsay AE, England RW: Computer analysis of serial electrocardiograms. Comput Biomed Res 5:709, 1972

6. Pipberger HV, McCaughan D, Littman D et al: Clinical application of a second generation electrocardiographic computer program. Am J Cardiol 35:597, 1975

7. Geddes JS, Warncr HR: A PVC detection program. Comput Biomed Res 4:493, 1971

8. Vetter NJ, Julian DG: Comparison of arrhythmia computer and conventional monitoring in coronary-care unit. Lancet 1:115 I, 1975

9. Jenkins JM: Automated electrocardiography and arrhythmia monitoring. Prog Cardiovasc Dis XXV:367, 1983 
10. Arzbaecher R: A pill electrode for the study of cardiac dysrhythmia. Medical Instrumentation, 12:277, 1978

11. Jenkins J, Wu D, Arzbaecher R: Computer diagnosis of supraventricular and ventricular arrhythmias. Circulation $60: 977,1979$

12. Arzbaecher R, Jenkins J, Collins S et al: Atrial electrical activity: the view from the esophagus. p. 314. IEEE/EMBS Conference (Frontiers of Engineering in Health Care), 1979

13. Lin D, Jenkins JM, DiCarlo LA: Analysis of P-wave changes on the esophageal electrogram: a two-lead arrhythmia analysis system using morphology and timing of both atrial and ventricular activation. Computers in cardiology. IEEE Press, New York, 1987

14. Feldman CL, Amazeen PG, Klein MD et al: Computer detection of ventricular ectopic beats. Comput Biomed Res 4:666, 1971

15. Arzbaecher R, Biancalana $P$, Stibolt $T$ et al: Computer technique for detection of cardiac dysrhythmias. Journal of the Association for the Advancement of Medical Instrumentation 5:104, 197 (Abstr)

16. Lin D, DiCarlo LA, Jenkins JM: Identification of ventricular tachycardia using intracavitary ventricular electrograms: Analysis of time and frequency domain patterns. PACE 11:1592, 1988

17. Throne $R$, Jenkins J, Winston $S$ et al: Discrimination of retrograde from anterograde atrial actrivation using intracardiac electrogram waveform analysis. PACE 12: 1622,1989

18. Throne R, DiCarlo LA, Jenkins $J$ et al: Paroxysmal bundle branch block of supraventricular origin: a possible source of misdiagnosis in detecting ventricular tachycardia using time domain analyses of intraventricular electrograms. PACE 13:453, 1990

19. Finelli $\mathrm{CJ}$, DiCarlo LA, Jenkins JM et al: Effects of heart rate and sympathetic tone on intraventricular electrogram morphology. Am J Cardiol 68:1321, 1991

20. DiCarlo LA, Jenkins JM, Winston SA, Kriegler C: Differentiation of ventricular tachycardia from ventricular fibrillation using intraventricular electrogram morphology. Am J Cardiol 70:820, 1992

21. Arzbaecher R, Collins S, Jenkins $J$ et al: Feasibility of long-term esophageal electrocardiography in the study of transient arrhythmias. Biomed Sci Instrum I 4: 1,1978

22. Manz M, Gerckens U, Luderitz B: Erroneous discharge from an implanted automatic cardioverter/defibrillator during supraventricular tachyarrhythmia-induced ventricular fibrillation. Am J Cardiol 7:343, 1986

23. Platia EV, Griffith LSC, Reid PR et al: Complications with the automatic implantable cardioverter defibrillator. J Am Col Card 2:200, 1986 (Abstr)

24. Steinberg JS, Sugalski JS, Haratonic K: Cardiac rhythm precipitating automatic-implantable cardioverter defibrillator discharge in outpatients. Am J Cardiol 67:95. 1991

25. DiCarlo LA, Throne RD, Jenkins JM: A time-domain analysis of intracardiac analysis electrograms for arrhythmia detection. PACE 14:329, 1991

26. Throne RD, Jenkins JM, DiCarlo LA: A comparison of four new time-domain techniques for discriminating ventricular tachycardia from sinus rhythm using ventricular waveform morphology. IEEE Trans Biomed Eng 38:561, 1991 


\section{Appendix}

\begin{tabular}{|c|c|c|c|c|c|c|c|c|c|}
\hline & \multicolumn{8}{|c|}{ Ladder diagrams, beat codes, and single beal diagnoses of AV sequence } & \multirow{2}{*}{$\begin{array}{l}0 \text { - short } \\
1 \text { - norma } \\
2 \text { - lono }\end{array}$} \\
\hline & $\mathrm{CCa}$ & $\mathrm{CCv}$ & A & AV & $w$ & $\mathrm{CCa}, \mathrm{CCv}=$ & $\begin{array}{l}0 \text { - abnormal } \\
1 \text { - normal }\end{array}$ & $A A \cdot A V, V V=$ & \\
\hline
\end{tabular}
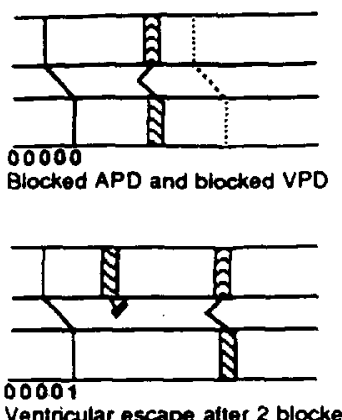

Ventricular escape after 2 blocked APDs
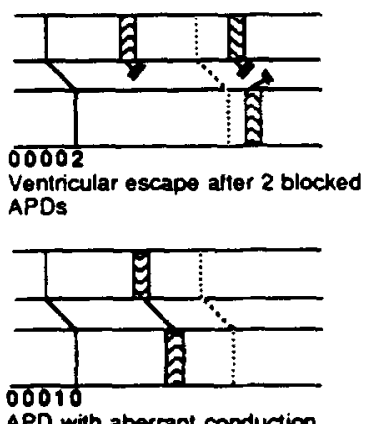

APD with aberrant conduction

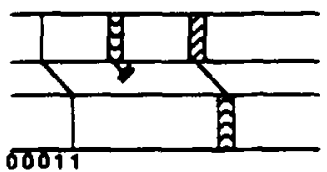

APD with aberrant conduction after blocked APD
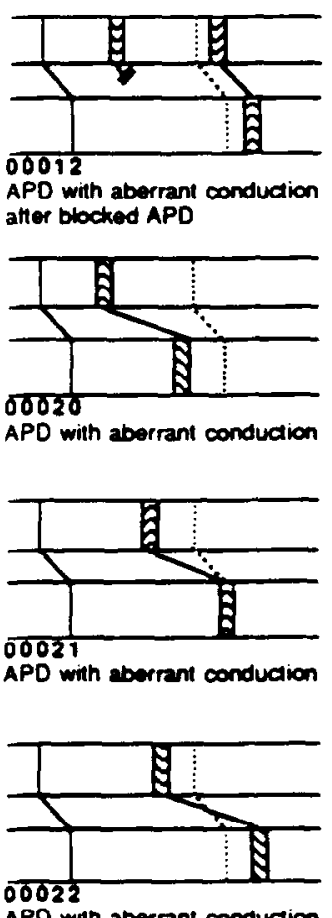

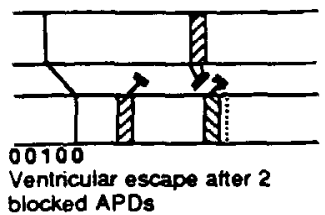

blocked APDs

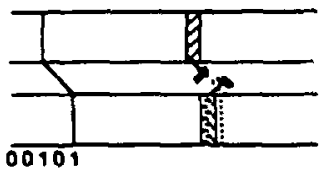

APD with aberrant conduction

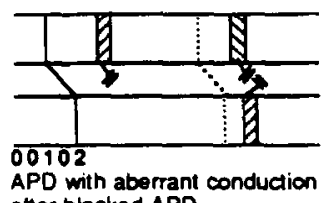

after blocked APD
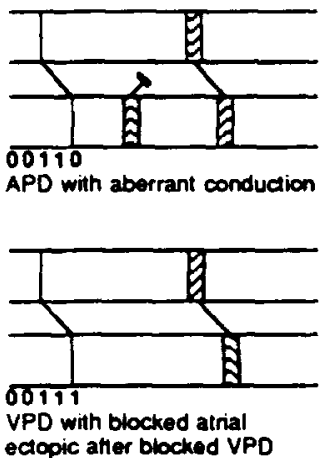

ectopic atter blocked VPD

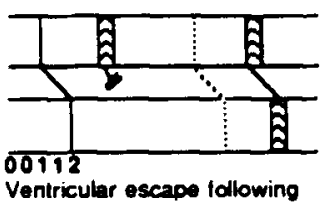

Ventricular escape following blocked APDs
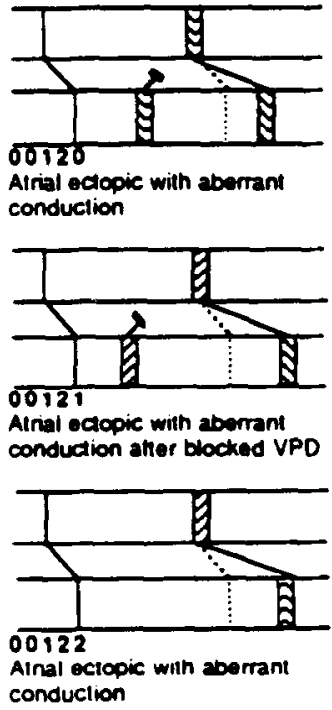

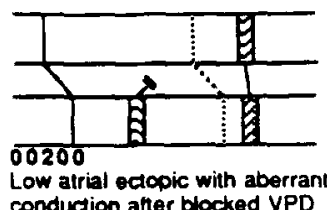

conduction after blocked VPD

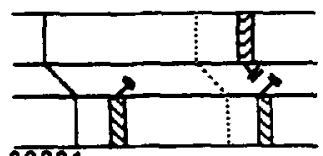

00201

Atrial escape and ventricular escape ather blocked VPD
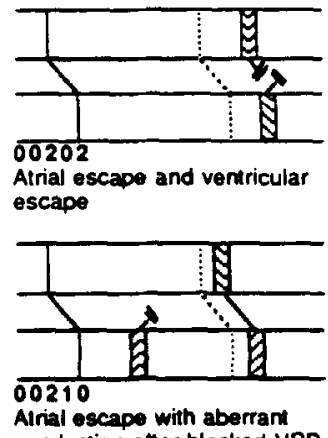

conduction atter blocked VPD
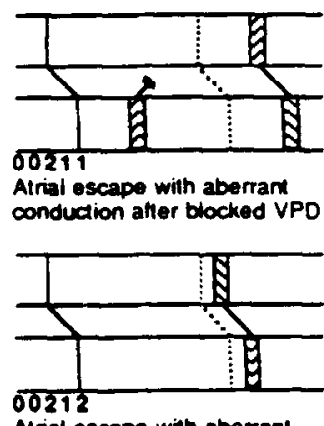

Alrial escape with aberrant conduction

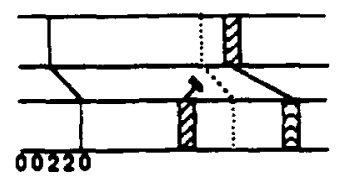

Alrial escape with aberrant conduction after blocked VPO
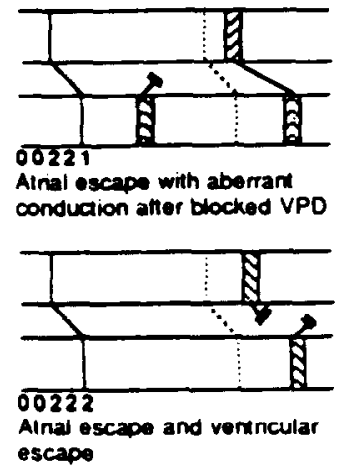
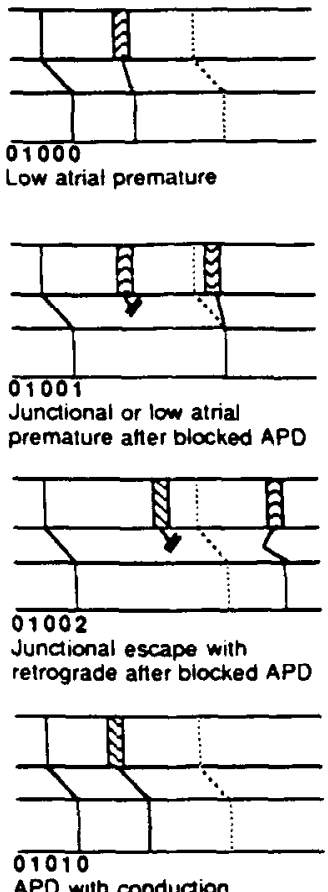

APO with conduction

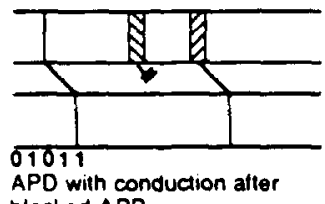

blocked APD
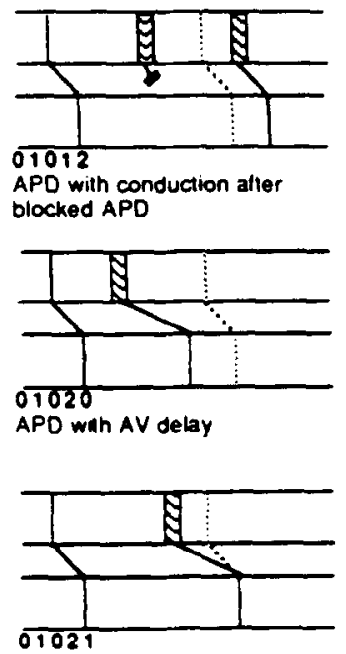

APD winh AV delay or weh junctional escape

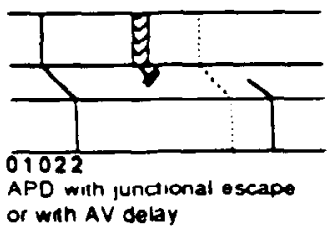



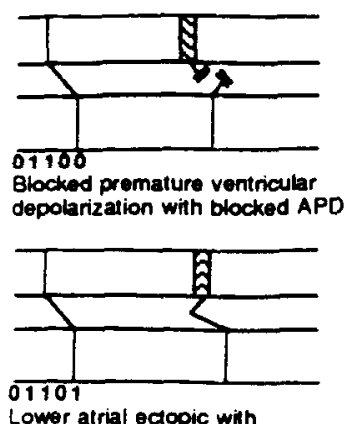

Lower atrial ectopic with normal conduction
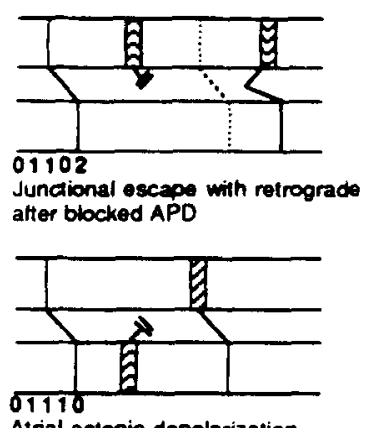

Atrial octopic depolarization after blocked VPD
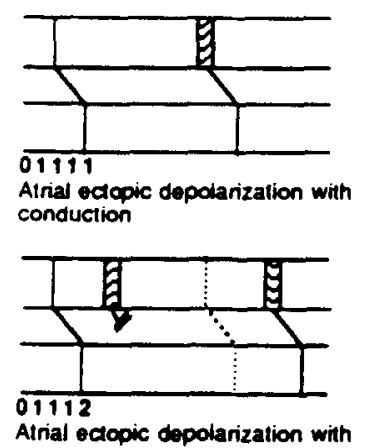
conduction after blocked APD
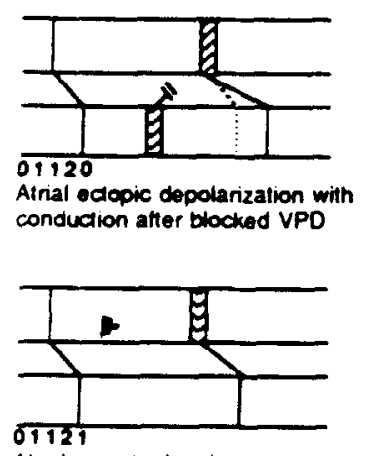

Alnal octopic depolarization with AV delay

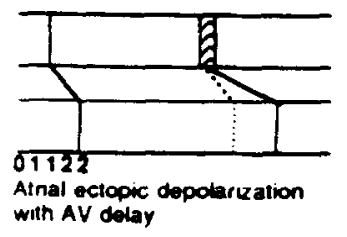

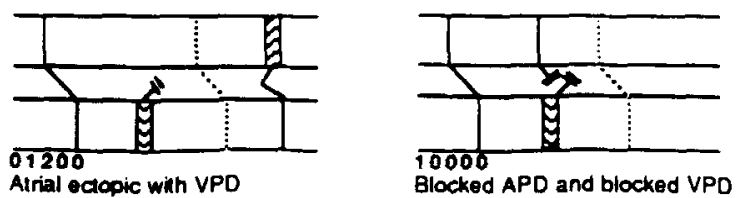

atter broch

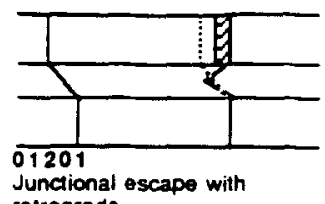

retrograde
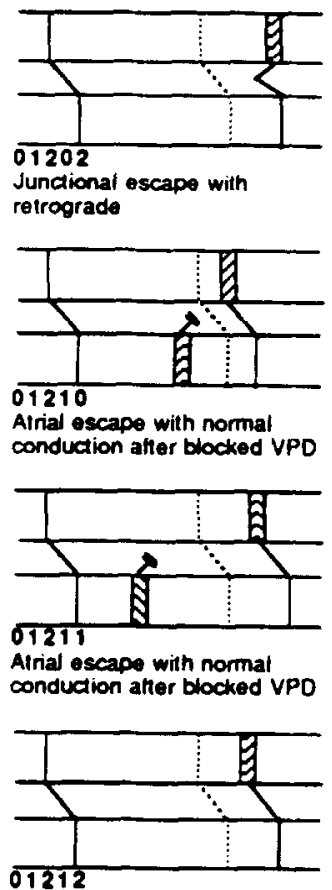

Atrial escape with conduction
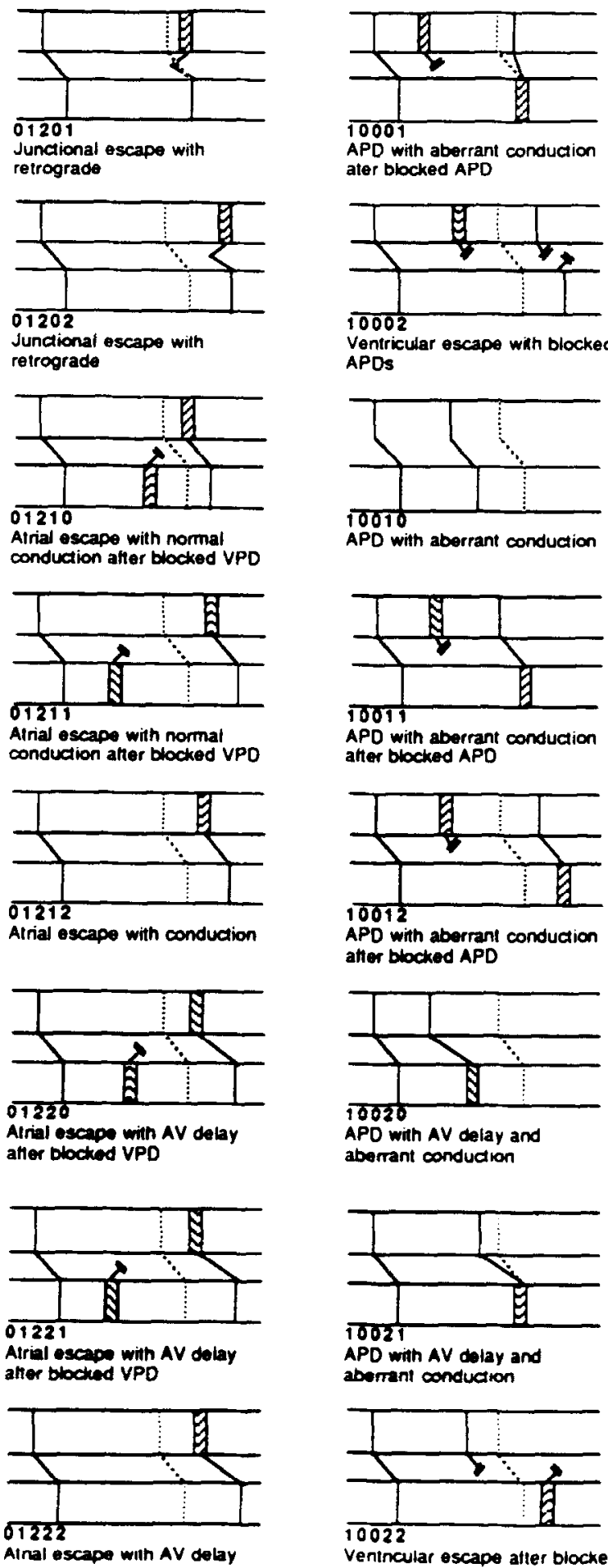
ater blocked APD
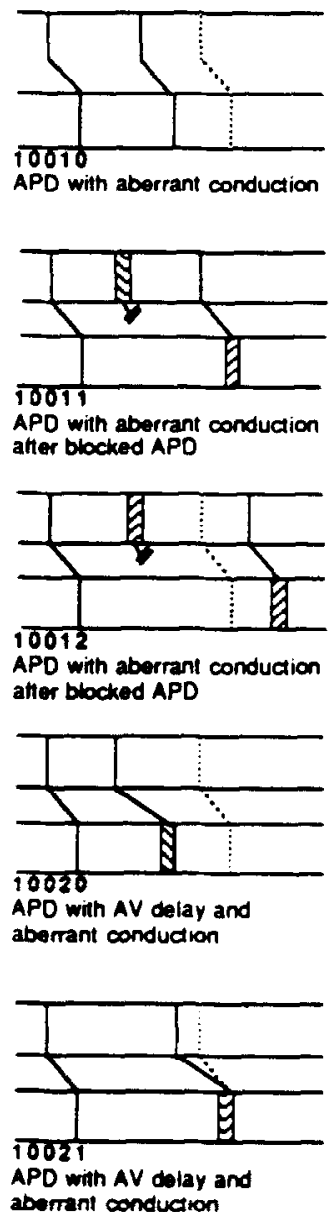
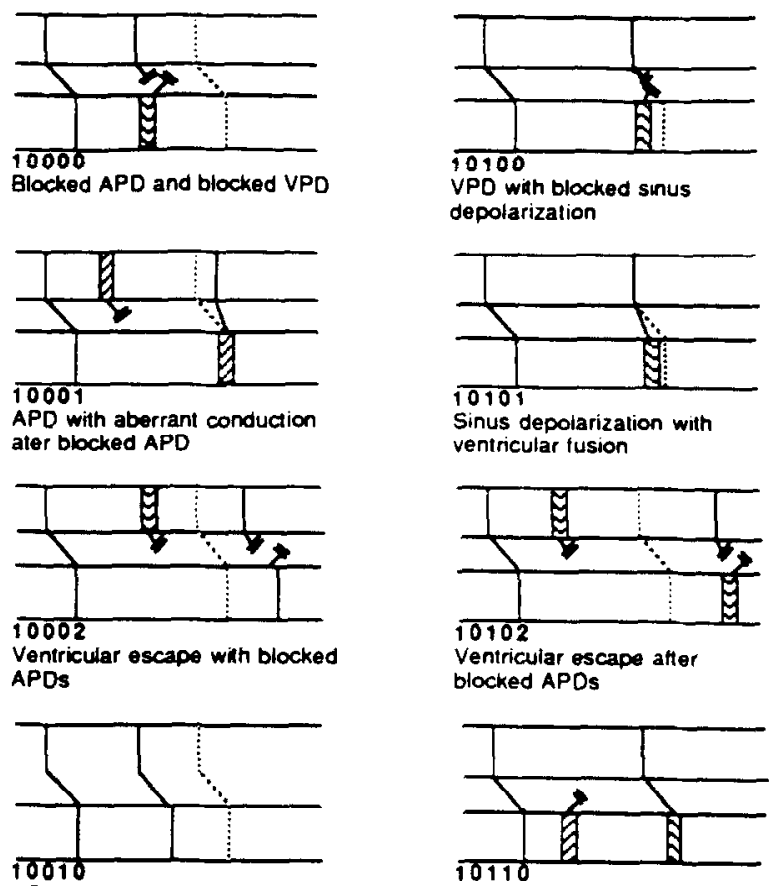

Sinus depolarization with aberran conduction after blocked VPD

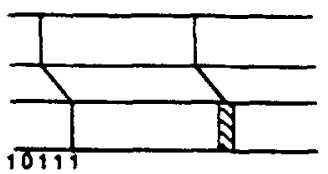

Sinus depolanzation with aberrant conduction

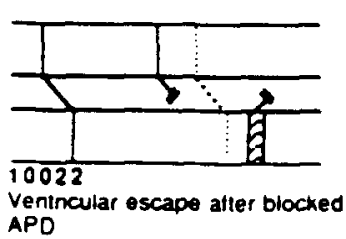

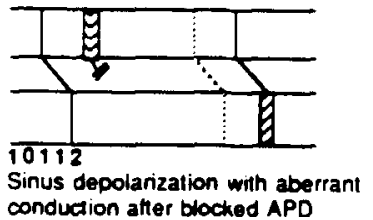

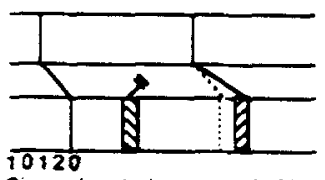

Sinus depolarization with AV delay and aberrant conduction atter blocked VPD

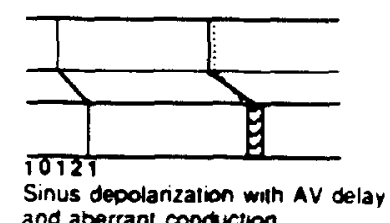
and aberrant conduction

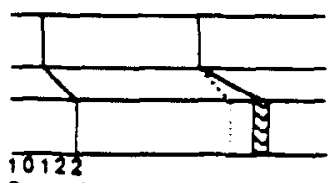

Sinus depolanzation with AV delay and aberrant conduction 

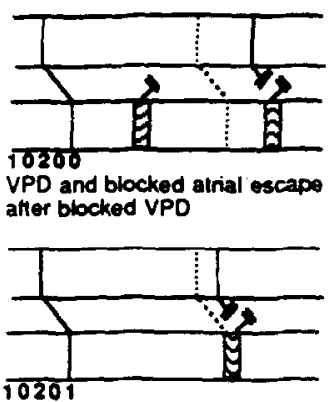

Sinus slowing with VPD without retrograde
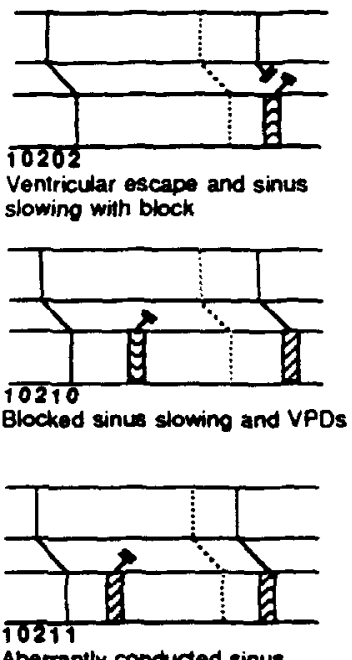

Aberrantly conducted sinus slowing aller blocked VPD

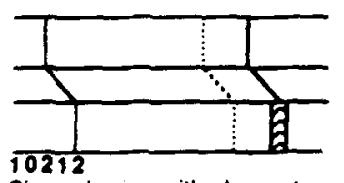

Sinus slowing with aberrant conduction
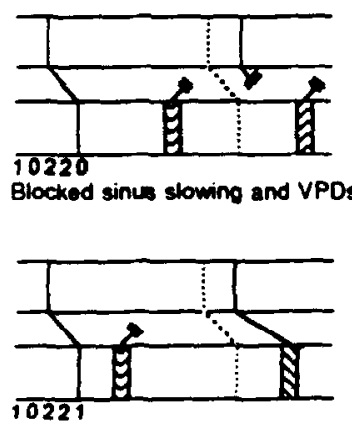

Sinus slowing with AV delay and aberrant conduction after biocked VPD

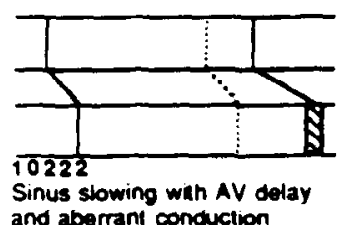

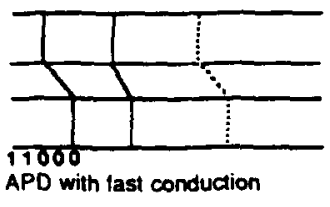

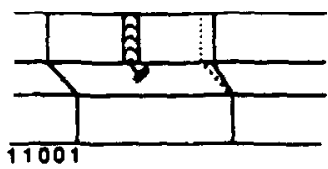

APD with fast conduction atter blocked APD

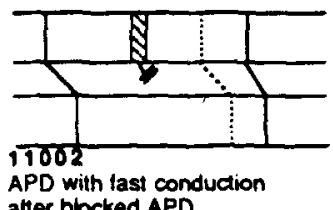
atter blocked APD
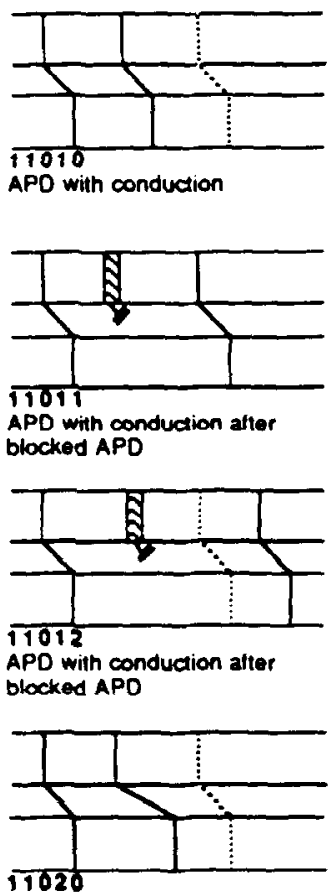

APD wah AV delay
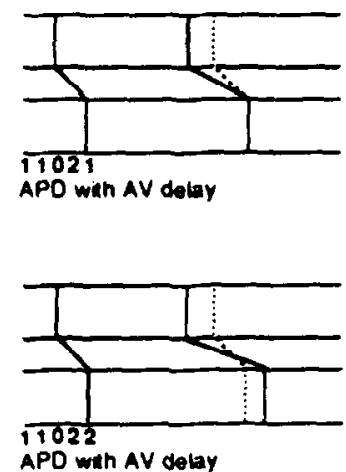

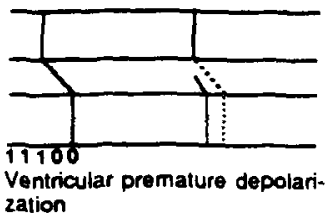
zation

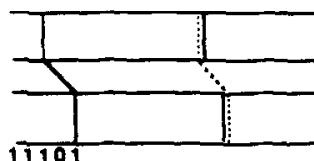

Sinus beat with block and junctiona escape of supemormal conduction

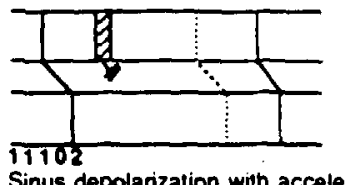

Sinus depolarization with acceleraled conduction atter blocked APD

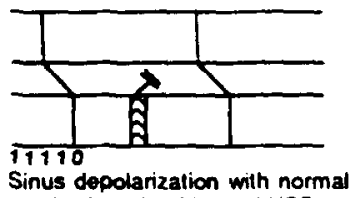

Sinus depolarization with no
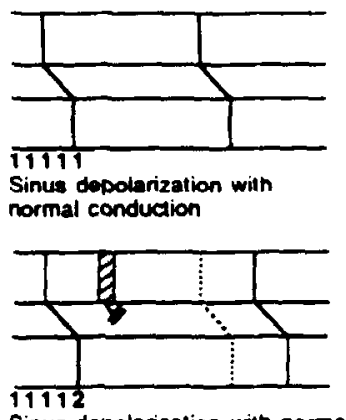

Sinus depolarization with normal conduction atter blocked APD
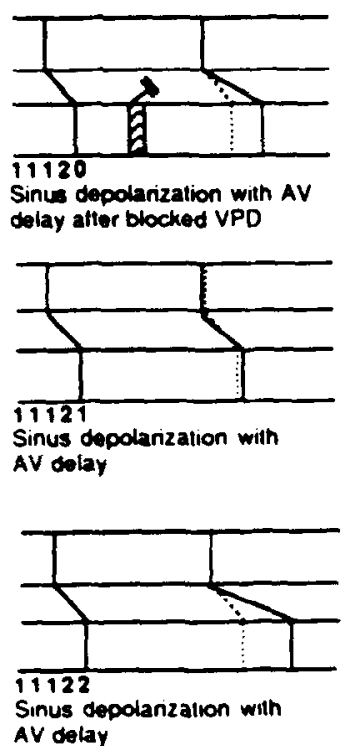
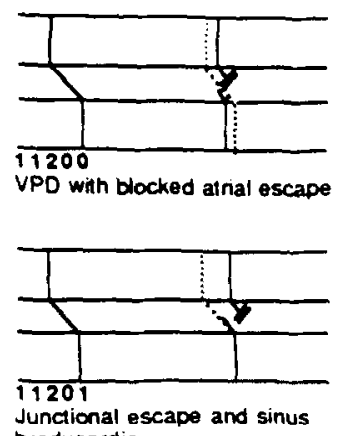

bradycardia

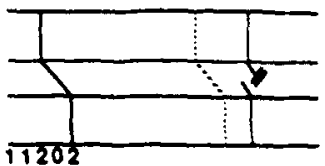

Sinus slowing with junctional escape

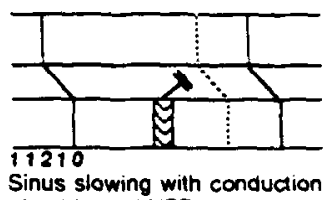
after blocked $V P D$

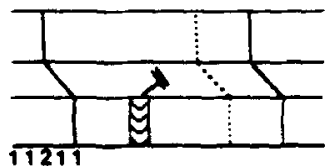
atter blocked VPD
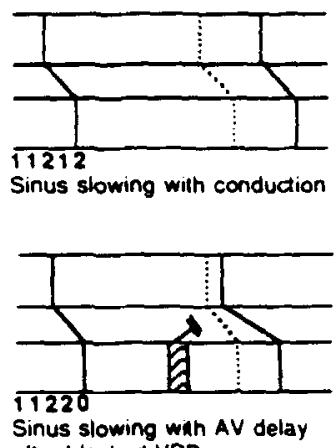
after blocked VPD
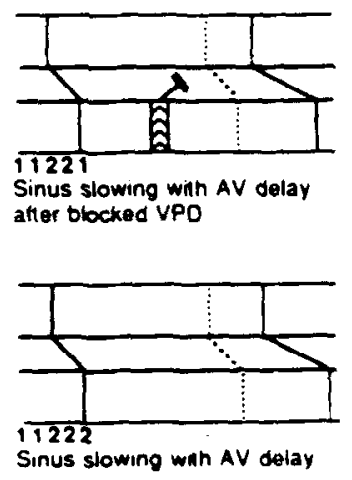
Automated Interpretation of Arrhythmias - DiCarlo et al. 67

Ladder diagrams, beat codes, and single beat diagnoses of VA sequence and blocked ventricular depolarization sequence

$$
\begin{array}{|l|l|}
\hline \operatorname{ccs} & \mathrm{v} v \\
\hline
\end{array}
$$

$c c v=0$-abnormal $W=0$-short

$\checkmark$ Sequence

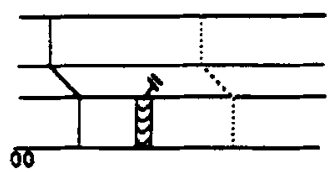

VPD without retrograde

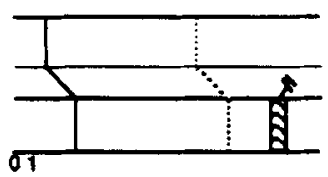

Venricular escape without retrograde

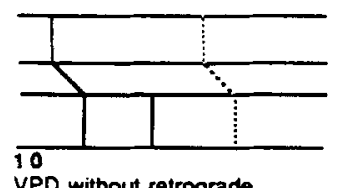

VPD without retrograde

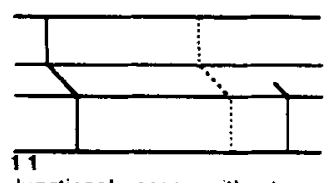

Junctional escape without retrograde

VA Sequence

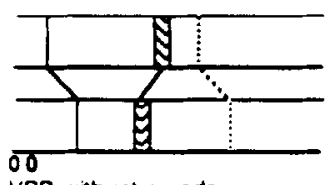

VPD with retrograde

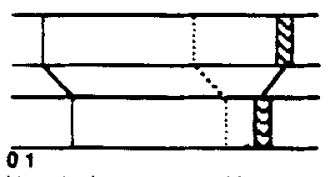

Ventricular escape with retrogrado
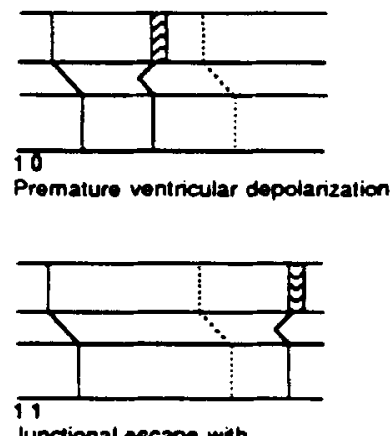

Junctional escape with retrograde
Laddet diagrams, beat codes, and single beat diagnoses of blocked atrial depolanization sequence

$$
\begin{array}{lll}
\hline C a & A A \\
\hline
\end{array}
$$

$$
\begin{aligned}
& \mathrm{CCa}=\begin{array}{l}
0-\text { abnormal } \\
1-\text { normal }
\end{array} \quad A A=1-\text { shon } \\
& 2 \text { - long }
\end{aligned}
$$

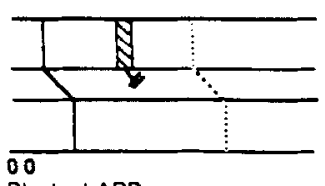

Blocked APD

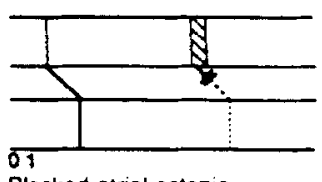

Blocked atrial ectopic

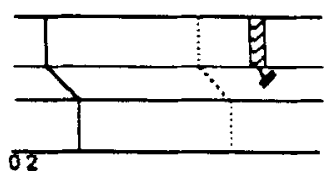

Blocked atrial escape

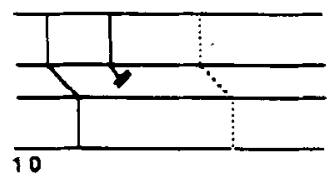

Blocked APD

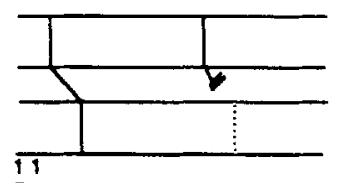

Blocked sinus depolanzation

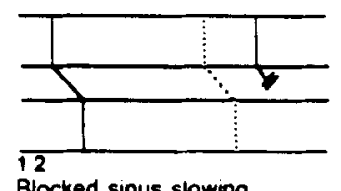

Blocked sinus slowing 